\title{
Dexmedetomidine and erythrocyte deformability
}

\author{
Sohn JT \\ Department of Anesthesiology and Pain Medicine, Gyeongsang National University College of Medicine, Gyeongsang National \\ University Hospital and Institute of Health Sciences, Gyeongsang National University, Jinju-si, Gyeongsangnam-do, Republic of \\ Korea.jtsohn@gnu.ac.kr
}

Text in PDF www.elis.sk.

Dear editor,

I read with interest the article entitled, "Effects of dexmedetomidine and thymoquinone on erythrocyte deformability in lower limb ischemia-reperfusion injury in streptozotocin-induced diabetic rats" recently published in Bratislava Medical Journal (1). The following comments could be considered while interpreting this article: First, erythrocyte deformability refers to the ability of the erythrocytes to change their shape under various pathophysiological conditions to improve perfusion (2). Although diabetes is known to decrease erythrocyte deformability index, which is associated with a decreased erythrocyte deformability, according to the data presented in this article, diabetes was found to increase erythrocyte deformability index $(1,2)$. In addition, dexmedetomidine and thymoquinone decreased the erythrocyte deformability index in rats with diabetes and ischemia-reperfusion injury (1). In other words, these results suggest that dexmedetomidine and thymoquinone adversely affect the ability of erythrocytes to change their shape in lower limb ischemia-reperfusion injury in diabetic rats. In the abstract of this article, the authors, however, have contradictorily mentioned that dexmedetomidine and thymoquinone may ameliorate the adverse effects of streptozotocin-induced lower limb ischemia-reperfusion injury in diabetic rats (1). It may cause some confusion for readers to interpret these data. Thus, the relationship between erythrocyte deformability index measured in this study and erythrocyte deformability should be described for readers to understand this study better. Second, thymoquinone, obtained from the seeds of Nigella sativa, has antioxidant effects. The maximum tolerated dose of intraperitoneal thymoquinone was reported to be $22.5 \mathrm{mg} / \mathrm{kg}$ in male rats (3). This study, however, used $25 \mathrm{mg} / \mathrm{kg}$ of intraperitoneal thymoquinone (1), which exceeded the maximum tolerated dose. In addition, thymoquinone was

Department of Anesthesiology and Pain Medicine, Gyeongsang National University College of Medicine, Gyeongsang National University Hospital, 15 Jinju-daero 816 beon-gil, Jinju-si, Gyeongsangnam-do 52727, Republic of Korea, and Institute of Health Sciences, Gyeongsang National University, Jinju-si, 52727, Republic of Korea

Address for correspondence: J.T. Sohn, Department of Anesthesiology and Pain Medicine, Gyeongsang National University Hospital, 79 Gangnam-ro, Jinju-si, 52727, Republic of Korea.

Phone: +82.55 .7508586 , Fax: +82.55 .7508142$ dissolved in dimethyl sulfoxide (DMSO, $0.5 \%$ ) and subsequently diluted in olive oil (3). As DMSO (0.2 to $0.6 \%$ ) induces hemolysis in vivo, it would have been more prudent to evaluate effects of the vehicle (DMSO and olive oil) alone on erythrocyte deformability in this experiment. Third, the highly selective alpha-2 adrenoceptor agonist dexmedetomidine has been widely used for sedation and attenuation of hemodynamic responses (4). Furthermore, dexmedetomidine enhances erythrocyte deformability in patients undergoing laparoscopic cholecystectomy, which seems to be partially mediated by the alpha-2 adrenoceptors (5). Thus, in order to elucidate the detailed mechanism, further investigations evaluating the effects of alpha-2 adrenoceptor blocker rauwolscine and yohimbine on dexmedetomidine-induced changes in erythrocyte deformability index are recommended in this study. I believe that this study would be a valuable report to suggest dexmedetomidineinduced enhanced erythrocyte deformability in lower limb ischemia reperfusion injury in streptozotocin-induced diabetic rats.

\section{References}

1. Ozer A, Comu FM, Kucuk A et al. Effects of dexmedetomidine and thymoquinone on erythrocyte deformability in lower limb ischemia reperfusion injury in streptozotocin-induced diabetic rats. Bratisl Med J 2018; 119 (10): 642-645.

2. Diamantopoulos EJ, Raptis SA, Moulopoulos SD. Red blood cell deformability index in diabetic retinopathy. Horm Metab Res 1987; 19 (11):569-573

3. Abukhader MM. The effect of route of administration in thymoquinone toxicity in male and female rats. Indian J Pharm Sci 2012; 74 (3): 195-200.

4. Surana P, Parikh DA, Patkar GA, Tendolkar BA. A prospective randomized controlled double-blind trial to assess the effects of dexmedetomidine during cleft palate surgery. Korean J Anesthesiol 2017; 70 (6): 633-641.

5. Yang XM, Liu J, Ji J, Xie J. Effects of dexmedetomidine on the deformability of erythrocytes in vitro and in anesthesia. Exp Ther Med 2014; 7 (6): 1631-1634.

Received December 24, 2018. Accepted February 5, 2019. 